\title{
Sparse normalized subband adaptive filter algorithm with
}

\section{$l_{0}$-norm constraint}

\author{
Yi Yu, Haiquan Zhao, Badong Chen
}

\begin{abstract}
In order to improve the filter's performance when identifying sparse system, this paper develops two sparse-aware algorithms by incorporating the $l_{0}$-norm constraint of the weight vector into the conventional normalized subband adaptive filter (NSAF) algorithm. The first algorithm is obtained from the principle of the minimum perturbation; and the second one is based on the gradient descent principle. The resulting algorithms have almost the same convergence and steady-state performance while the latter saves computational complexity. What's more, the performance of both algorithms is analyzed by resorting to some assumptions commonly used in the analyses of adaptive algorithms. Simulation results in the context of sparse system identification not only demonstrate the effectiveness of the proposed algorithms, but also verify the theoretical analyses.
\end{abstract}

Keywords: Normalized subband adaptive filter, sparsity attraction, $l_{0}$-norm, sparse system identification.

\section{Introduction}

Adaptive filtering algorithm is a very important branch in modern signal processing fields, and has had a wide range of applications such as system identification, active noise cancellation, channel estimation, and echo cancellation [1-3, 33-36]. Compared with the well-known normalized least mean square (NLMS) algorithm, the family of subband adaptive filter (SAF) algorithm exhibits faster convergence when the input signal is colored (also called the correlated input signal). In all the SAFs, the colored input signal is divided into multiple subband signals; and each decimated subband input signal used for updating the weights is approximately white, thus enhancing the convergence rate of filter [2]. In [4], Lee and Gan proposed the normalized SAF (NSAF) algorithm based on the multiband-structure of SAF, which converges faster than the NLMS for the colored inputs. Also, the NSAF has almost the same computational complexity as the NLMS for a long filter. Hence, one of the interesting applications of NSAF is echo cancellation. Following the NSAF, researchers have done many works in terms of analyzing and improving the performance of NSAF [5-10]. For instance, to overcome a tradeoff between fast convergence rate and low steady-state error, several variable step size NSAF algorithms were developed from different criteria [8-10].

In practical scenarios, one often encounters many systems with sparse property, that is, there are only a small number of nonzero entries in its impulse response. Examples of such system include the echo paths [11] and digital TV transmission channels [12]. The sparsity of the underlying system if exploited properly can lead to a significant improvement in the identification performance of adaptive algorithm. The original adaptive filter algorithms such as the NLMS and the NSAF will suffer from slow convergence when the system to be identified is sparse, since they do not make use of the sparsity of system. To overcome this limitation, one of the effective approaches is the so-called proportionate NLMS algorithms [13-16]. For this type of algorithms, the fundamental concept is to assign an independent step size for each filter coefficient which is proportional to the magnitude of that coefficient, thus resulting in fast convergence rate for identifying sparse system. Likewise, to speed up the convergence of NSAF in a sparse system, Abadi et al. developed its proportionate family by directly extending

Yi Yu and Haiquan Zhao are with the School of Electrical Engineering at Southwest Jiaotong University, Chengdu, 610031, China.

Badong Chen is with the School of Electronic and Information Engineering, Xi'an Jiaotong University, Xi'an,China.

E-mail: yuyi_xyuan@163.com (Y.YY), hqzhao_swjtu@126.com (H. Zhao), chenbd@mail.xjtu.edu.cn (B. Chen).

Corresponding author

1

(C) 2016. This manuscript version is made available under the Elsevier user license

http://www.elsevier.com/open-access/userlicense/1.0/ 
the proportionate ideas presented in the NLMS to the NSAF [17].

Recently, motivated by the LASSO [18] and compressive sensing framework [19], an alternative approach to deal with sparsity has been studied, where a sparsity-promoting norm (e.g., the $l_{0}$ - and $l_{1}$-norms) of the weight vector is added to the original cost function. Up to present, based on these sparsity-promoting norms and their improvements, a large number of sparse adaptive algorithms have been developed [20-32]. In [20], a called zero-attraction LMS (ZA-LMS) algorithm was derived by incorporating the $l_{1}$-norm of the weight vector into the cost function of the standard LMS, which obtains better steady-state performance than the LMS in a sparse system. And, to further improve the performance, the authors also proposed a reweighted ZA-LMS (RZA-LMS) algorithm. At the same time, Gu et al. embedded the $l_{0}$-norm into the LMS's cost function, yielding the $l_{0}$-LMS algorithm $[21,22]$ which performs better than the LMS in both the convergence rate and steady-state error when the system is sparse. Benefited from the successes of sparsity-promoting norms in the LMS, similar ways have also been applied to other types of adaptive algorithms such as the sparse affine projection algorithms [23, 24, 32], sparse recursive least square (RLS) algorithms [25, 26], and sparse distributed filtering algorithms [27]. Likewise, for the sake of improving the performance of NSAF in sparse scenarios, Choi developed the $l_{1}$-norm-based NSAF ( $l_{1}$-NSAF) algorithm and its reweighted version $\left(l_{1}\right.$-RNSAF) [28]. To the best of our knowledge, however, the application of the $l_{0}$-norm in the NSAF has not been studied.

Considering the $l_{0}$-norm can better measure the sparsity of the underlying system than the $l_{1}$-norm, in this paper, we will focus on developing two sparse NSAF algorithms by introducing the $l_{0}$-norm into the NSAF's optimization function so as to improve the performance of the NSAF in sparse environments. In particular, our main contributions are listed as follows:

1. Two $l_{0}$-norm-based NSAF algorithms are proposed, which are derived from the principle of the minimum perturbation and the gradient descent method, respectively. These two algorithms have similar performance while the latter reduces the computational complexity.

2. The convergence of both proposed algorithms and their superiority over the NSAF are analyzed, which are also supported by simulations.

3. Simulations in various sparse systems are presented to verify the advantages of our proposed algorithms relative to some existing algorithms.

\section{Review NSAF algorithm}

The desired signal $d(n)$ that obtains from the output-end of the unknown system is formulated by

$$
d(n)=\mathbf{u}^{T}(n) \mathbf{w}_{o}+\eta(n)
$$

where $\mathbf{w}_{o}$ is an unknown $M$-dimensional sparse vector to be estimated, $\mathbf{u}(n)=[u(n), u(n-1), \ldots, u(n-M+1)]^{T}$ denotes the input signal vector, $\eta(n)$ is the background noise, and $(\cdot)^{T}$ indicates the transpose. Fig. 1 shows the block diagram of multiband-structured SAF, where $N$ denotes number of subbands. The desired signal $d(n)$ and the input signal $u(n)$ are partitioned into multiple subband signals $d_{i}(n)$ and $u_{i}(n)$ through the analysis filter bank $\left\{H_{i}(z), i=0,1, \ldots, N-1\right\}$, respectively. The subband output signal $y_{i}(n)$ is obtained by filtering $u_{i}(n)$ through an adaptive filter whose weight vector is denoted as $\mathbf{w}(k)=\left[w_{1}(k), w_{2}(k), \ldots, w_{M}(k)\right]^{T}$. Then, the signals $y_{i}(n)$ and $d_{i}(n)$ are $N$-fold decimated to yield the subband signals $y_{i, D}(k)$ and $d_{i, D}(k)$ with a lower sampling rate, respectively, where $y_{i, D}(k)=\mathbf{u}_{i}^{T}(k) \mathbf{w}(k)$, $\mathbf{u}_{i}(k)=\left[u_{i}(k N), u_{i}(k N-1), \ldots, u_{i}(k N-M+1)\right]^{T}$ and $d_{i, D}(k)=d_{i}(k N)$. Here, we use $n$ and $k$ to indicate the original sequences and decimated sequences, respectively. The subband error signals $e_{i, D}(k)$ for $i=0,1, \ldots, N-1$ are obtained as 


$$
e_{i, D}(k)=d_{i, D}(k)-y_{i, D}(k)=d_{i, D}(k)-\mathbf{u}_{i}^{T}(k) \mathbf{w}(k) .
$$

As reported in [4], the standard NSAF algorithm is expressed as

$$
\mathbf{w}(k+1)=\mathbf{w}(k)+\mu \sum_{i=0}^{N-1} \frac{e_{i, \mathrm{D}}(k) \mathbf{u}_{i}(k)}{\left\|\mathbf{u}_{i}(k)\right\|_{2}^{2}}
$$

where $\mu \quad(0<\mu<2)$ is the step-size and $\|\cdot\|_{2}$ denotes the $l_{2}$-norm of a vector.

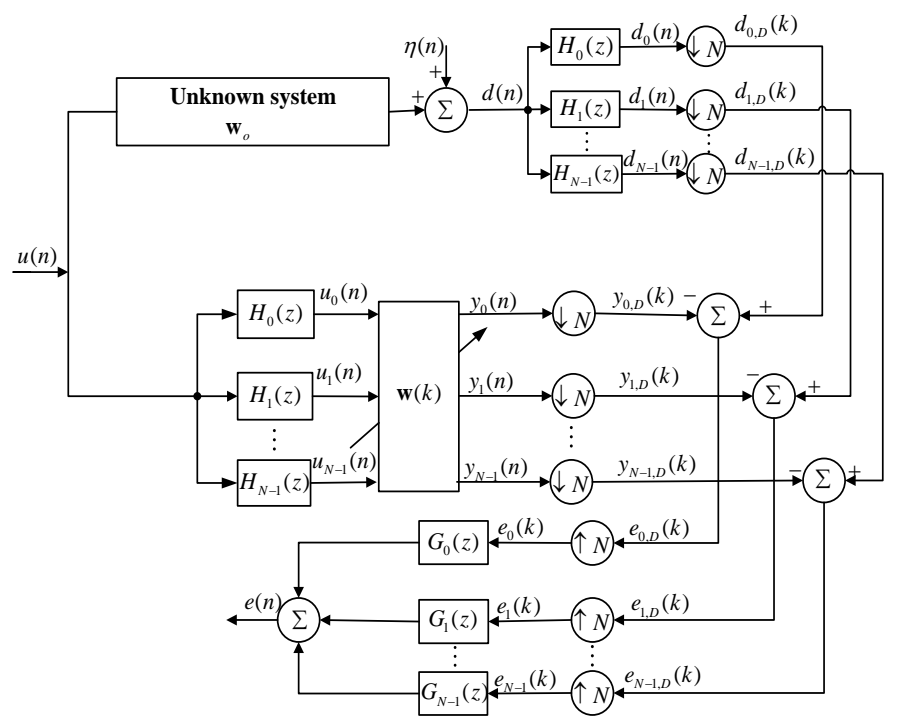

Fig. 1. Block diagram of multiband-structured SAF.

\section{Proposed algorithms}

Benefited from the feature of the $l_{0}$-norm that directly quantifies the sparsity of a vector, in this section, we develop two zero-norm-based NSAF (ZN-NSAF) algorithms, named as the ZN-NSAF-I and ZN-NSAF-II, respectively.

\subsection{Derivation of ZN-NSAF-I}

To derive the ZN-NSAF-I, a minimum perturbation problem that involves the $l_{0}$-norm of the updated weight vector $\mathbf{w}(k+1)$ is defined as

$$
\begin{aligned}
& \min _{\mathbf{w}(k+1)}\left\{\|\mathbf{w}(k+1)-\mathbf{w}(k)\|_{2}^{2}+\rho\|\mathbf{w}(k+1)\|_{0}\right\} \text { subject to } \\
& d_{i, D}(k)-\mathbf{u}_{i}^{T}(k) \mathbf{w}(k+1)=0, i=0,1, \ldots, N-1
\end{aligned}
$$

where \|\|$_{0}$ denotes the $l_{0}$-norm of a vector that counts the number of nonzero entries $[20,21]$, and $\rho>0$ controls the intensity given to the $l_{0}$-norm attraction term. To solve the optimization problem (4), we form the Lagrangian function as

$$
J_{1}(k)=\|\mathbf{w}(k+1)-\mathbf{w}(k)\|_{2}^{2}+\rho\|\mathbf{w}(k+1)\|_{0}+\sum_{i=0}^{N-1}\left(d_{i, D}(k)-\mathbf{u}_{i}^{T}(k) \mathbf{w}(k+1)\right) \lambda_{i}
$$

where $\lambda_{i}$ is the Lagrange multiplier. Set the derivative of $J_{1}(k)$ with respect to $\mathbf{w}(k+1)$ to be zero, we have

$$
\mathbf{w}(k+1)=\mathbf{w}(k)+\frac{1}{2} \sum_{i=0}^{N-1} \mathbf{u}_{i}(k) \lambda_{i}-\frac{1}{2} \rho \nabla\|\mathbf{w}(k+1)\|_{0}
$$

where $\nabla\|\mathbf{w}(k+1)\|_{0} \triangleq \partial\|\mathbf{w}(k+1)\|_{0} / \partial \mathbf{w}(k+1)$. Substituting (6) into the constraints in (4), $\lambda_{i}, \quad i=0,1, \ldots, N-1$ are obtained as

$$
\lambda_{i}=\frac{2 e_{i, \mathrm{D}}(k)}{\left\|\mathbf{u}_{i}(k)\right\|_{2}^{2}}+\rho \frac{\mathbf{u}_{i}^{T}(k)}{\left\|\mathbf{u}_{i}(k)\right\|_{2}^{2}} \nabla\|\mathbf{w}(k+1)\|_{0} .
$$

Notice that in derivation of (7) we also use the diagonal assumption, i.e., $\mathbf{u}_{i}^{T}(k) \mathbf{u}_{j}(k) \approx 0, i \neq j$, which has been used for 
obtaining the NSAF [4]. Inserting (7) into (6) and introducing the step size $\mu$, the update of the weight vector of the algorithm is formulated as

$$
\mathbf{w}(k+1)=\mathbf{w}(k)+\mu \sum_{i=0}^{N-1} \frac{e_{i, \mathrm{D}}(k) \mathbf{u}_{i}(k)}{\left\|\mathbf{u}_{i}(k)\right\|_{2}^{2}}+\frac{1}{2} \mu \rho\left(\sum_{i=0}^{N-1} \frac{\mathbf{u}_{i}(k) \mathbf{u}_{i}^{T}(k)}{\left\|\mathbf{u}_{i}(k)\right\|_{2}^{2}}-\mathbf{I}\right) \nabla\|\mathbf{w}(k)\|_{0}
$$

where $\mathbf{I}$ is a unit matrix of size $M \times M$. In (8), we also use a replacement of $\nabla\|\mathbf{w}(k+1)\|_{0}$ with $\nabla\|\mathbf{w}(k)\|_{0}$, since $\mathbf{w}(k+1)$ is not available at the current $k$ iteration.

\subsection{Derivation of ZN-NSAF-II}

The ZN-NSAF-II is obtained by minimizing a new cost function that includes the cost function of the standard NSAF [7] and the $l_{0}$-norm attraction of the current weight vector which is formulated as

$$
J_{2}(k)=\frac{1}{2} \sum_{i=0}^{N-1}\left(\frac{e_{i, \mathrm{D}}(k)}{\left\|\mathbf{u}_{i}(k)\right\|_{2}}\right)^{2}+\frac{1}{2} \rho\|\mathbf{w}(k)\|_{0}
$$

Applying the gradient descent method in order to minimize (12), the weight vector of the ZN-NSAF-II is updated as

$$
\begin{aligned}
\mathbf{w}(k+1) & =\mathbf{w}(k)-\mu \frac{\partial J_{2}(k)}{\partial \mathbf{w}(k)} \\
& =\mathbf{w}(k)+\mu \sum_{i=0}^{N-1} \frac{e_{i, \mathrm{D}}(k) \mathbf{u}_{i}(k)}{\left\|\mathbf{u}_{i}(k)\right\|_{2}^{2}}-\frac{1}{2} \mu \rho \nabla\|\mathbf{w}(k)\|_{0}
\end{aligned}
$$

\subsection{Calculation of $\nabla\|\mathbf{w}(k)\|_{0}$}

To implement the above ZN-NSAF-I and ZN-NSAF-II algorithms, now, the only problem that remains is how to compute $\nabla\|\mathbf{w}(k)\|_{0}$. Since the minimization of the $l_{0}$-norm is a Non-Polynomial (NP) hard problem, $\nabla\|\mathbf{w}(k)\|_{0}$ is usually approximated by the following piecewise function (see [20, 21] for details)

$$
\begin{gathered}
\nabla\|\mathbf{w}(k)\|_{0} \triangleq\left[f_{\beta}\left(w_{1}(k)\right), f_{\beta}\left(w_{2}(k)\right), \ldots, f_{\beta}\left(w_{M}(k)\right)\right]^{T}, \\
f_{\beta}\left(w_{m}(k)\right) \approx\left\{\begin{array}{cc}
-\beta^{2} w_{m}(k)-\beta, & -1 / \beta \leq w_{m}(k)<0 \\
-\beta^{2} w_{m}(k)+\beta, & 0<w_{m}(k) \leq 1 / \beta \\
0 & \text { elsewhere }
\end{array}\right.
\end{gathered}
$$

where $\beta$ is a positive constant.

Remark 1: The last term in (8) and/or (10) is called the zero-attractor whose role is to impose an attraction to zero on small weights, thereby speeding up convergence. Note that only the weights within the attraction region given by $[-1 / \beta, 1 / \beta]$ are attracted. And, in this region, the closer the weight is to zero, the greater the attraction intensity is. If the weight is not in this region, no attraction will be performed. Since the near-zero coefficients in sparse system identification are in the majority, both proposed algorithms can provide fast convergence rate. In addition, a larger $\beta$ leads to stronger attraction intensity, but also leads to a narrower attraction region. However, it is difficult to evaluate the effect of $\beta$ on the performance of algorithm in theory. In practice, according to our extensive simulation results, $\beta \in[5,20]$ can result in good performance.

Remark 2: The last term in (8) considers the projection of $\nabla\|\mathbf{w}(k)\|_{0}$ onto the subspace which is orthogonal to the space spanned by $\left\{\mathbf{u}_{i}(k), i=0,1, \ldots, N-1\right\}$. This property ensures that the subband posterior errors of the ZN-NSAF-I are always equal to zero at each iteration when $\mu=1$, i.e., satisfying the subband constraints in (4). The ZN-NSAF-II directly uses 
$\nabla\|\mathbf{w}(k)\|_{0}$ to update the weight vector, see (10), thus its subband posterior errors are not zero, i.e., $d_{i, D}(k)-\mathbf{u}_{i}{ }^{T}(k) \mathbf{w}(k+1)=\frac{\rho}{2} \mathbf{u}_{i}^{T}(k) \nabla\|\mathbf{w}(k)\|_{0} \neq 0$ for each subband. Based on the above reasons, the performance of the ZN-NSAF-I should be superior to that of the ZN-NSAF-II. Practically, this advantage can be ignored thorough our simulations due mainly to the fact that the value of $\rho$ is very small. It is noticeable that as compared to the ZN-NSAF-I, the ZN-NSAF-II reduces $2 M^{2} N+M^{2}$ multiplications and $M^{2} N$ additions for per iteration, since it does not require the calculation of $\left(\sum_{i=0}^{N-1} \mathbf{u}_{i}(k) \mathbf{u}_{i}^{T}(k) /\left\|\mathbf{u}_{i}(k)\right\|_{2}^{2}\right) \nabla\|\mathbf{w}(k)\|_{0}$.

\section{Performance analyses}

In this section, we analyze the performance of the ZN-NSAF-I and ZN-NSAF-II algorithms in a unified manner. Before starting, both algorithms are unified as

$$
\mathbf{w}(k+1)=\mathbf{w}(k)+\mu \sum_{i=0}^{N-1} \frac{e_{i, \mathrm{D}}(k) \mathbf{u}_{i}(k)}{\left\|\mathbf{u}_{i}(k)\right\|_{2}^{2}}-\frac{1}{2} \mu \rho \mathbf{P}(k) \mathbf{f}_{\beta}(\mathbf{w}(k))
$$

where the ZN-NSAF-I and ZN-NSAF-II are obtained by letting $\mathbf{P}(k)=\mathbf{I}-\sum_{i=0}^{N-1} \mathbf{u}_{i}(k) \mathbf{u}_{i}^{T}(k) /\left\|\mathbf{u}_{i}(k)\right\|_{2}^{2}$ and $\mathbf{P}(k)=\mathbf{I}$, respectively. For convenience of analysis, some assumptions are necessary.

Assumption 1: The background noise $\eta(n)$ is a zero-mean white Gaussian process with variance $\sigma_{\eta}^{2}$.

Assumption 2: The analysis filter bank used for partitioning the signals $u(n)$ and $d(n)$ is paraunitary [5, 6]. Hence, we have

$$
d_{i, D}(k)=\mathbf{u}_{i}^{T}(k) \mathbf{w}_{o}+\eta_{i, D}(k)
$$

where the subband noises $\eta_{i, D}$ for $i=0,1, \ldots, N-1$ are also zero-mean white Gaussian but variance $\sigma_{\eta_{i}}^{2}=\sigma_{\eta}^{2} / N \quad[6]$.

Assumption 3: $\mathbf{w}(k), \mathbf{u}_{i}(k)$, and $\eta_{i}(k)$ are statistically independent. This is so called independence assumption [1, 6].

Assumption 4: Each decimated subband input signal is approximately white, i.e., $E\left[\mathbf{u}_{i}(k) \mathbf{u}_{i}^{T}(k)\right] \approx \sigma_{u_{i}}^{2}(k) \mathbf{I}$ and $E\left[\mathbf{u}_{i}^{T}(k) \mathbf{u}_{i}(k)\right] \approx M \sigma_{u_{i}}^{2}(k)[2,9]$, where $E[\cdot]$ denotes the expectation.

Assumption 5: For a long filter, i.e., $M \gg 1$, the fluctuation of $\left\|\mathbf{u}_{i}(k)\right\|_{2}^{2}$ from one iteration to the next is small enough.

\subsection{Convergence of both algorithms}

Subtracting (14) from $\mathbf{w}_{o}$, we have

$$
\tilde{\mathbf{w}}(k+1)=\tilde{\mathbf{w}}(k)-\mu \sum_{i=0}^{N-1} \frac{e_{i, \mathrm{D}}(k) \mathbf{u}_{i}(k)}{\left\|\mathbf{u}_{i}(k)\right\|_{2}^{2}}+\frac{1}{2} \mu \rho \mathbf{P}(k) \mathbf{f}_{\beta}(\mathbf{w}(k)),
$$

where $\tilde{\mathbf{w}}(k) \triangleq \mathbf{w}_{o}-\mathbf{w}(k)$ denotes the weight error vector. Taking the squared $l_{2}$-norm and mathematical expectation of both sides of (15), the following relation is founded:

$$
\begin{aligned}
\operatorname{MSD}(k+1)= & \operatorname{MSD}(k)+\left\{\mu E\left[\tilde{\mathbf{w}}^{T}(k) \mathbf{P}(k) \mathbf{f}_{\beta}(\mathbf{w}(k))\right]-\mu^{2} E\left[\sum_{i=0}^{N-1} \frac{e_{i, \mathrm{D}}(k) \mathbf{u}_{i}{ }^{T}(k)}{\left\|\mathbf{u}_{i}(k)\right\|_{2}^{2}} \mathbf{P}(k) \mathbf{f}_{\beta}(\mathbf{w}(k))\right]\right\} \\
& +\frac{1}{4} \mu^{2} E\left[\left\|\mathbf{P}(k) \mathbf{f}_{\beta}(\mathbf{w}(k))\right\|^{2}\right] \rho^{2}-2 \mu E\left[\sum_{i=0}^{N-1} \frac{\tilde{\mathbf{w}}^{T}(k) \mathbf{u}_{i}(k) e_{i, \mathrm{D}}(k)}{\left\|\mathbf{u}_{i}(k)\right\|_{2}^{2}}\right]+\mu^{2} E\left[\sum_{i=0}^{N-1} \frac{e_{i, \mathrm{D}}^{2}(k)}{\left\|\mathbf{u}_{i}(k)\right\|_{2}^{2}}\right]
\end{aligned}
$$


where $\operatorname{MSD}(k) \triangleq E\left[\|\tilde{\mathbf{w}}(k)\|^{2}\right]$ represents the mean square deviation (MSD). In (16), we again use the previous diagonal assumption. According to (16), we define a bivariate function with respect to $\mu$ and $\rho$ as

$$
h(\mu, \rho)=\operatorname{MSD}(k+1)-\operatorname{MSD}(k)=a(k) \rho^{2}+b(k) \rho+c(k)
$$

where $a(k)=\frac{1}{4} \mu^{2} E\left[\left\|\mathbf{P}(k) \mathbf{f}_{\beta}(\mathbf{w}(k))\right\|^{2}\right], \quad c(k)=\mu^{2} E\left[\sum_{i=0}^{N-1} e_{i, \mathrm{D}}^{2}(k) /\left\|\mathbf{u}_{i}(k)\right\|_{2}^{2}\right]-2 \mu E\left[\sum_{i=0}^{N-1} \tilde{\mathbf{w}}^{T}(k) \mathbf{u}_{i}(k) e_{i, \mathrm{D}}(k) /\left\|\mathbf{u}_{i}(k)\right\|_{2}^{2}\right]$, and $b(k)=\mu E\left[\tilde{\mathbf{w}}^{T}(k) \mathbf{P}(k) \mathbf{f}_{\beta}(\mathbf{w}(k))\right]-\mu^{2} E\left[\left(\sum_{i=0}^{N-1} e_{i, \mathrm{D}}(k) \mathbf{u}_{i}{ }^{T}(k) /\left\|\mathbf{u}_{i}(k)\right\|_{2}^{2}\right) \mathbf{P}(k) \mathbf{f}_{\beta}(\mathbf{w}(k))\right]$. For ensuring the convergence of the proposed algorithms in mean square sense, the MSD must monotonically decrease as the iteration goes on, i.e., $h(\mu, \rho)<0$. Consider the fact the proposed algorithms should work properly even if in a particular case that the sparsity of the underlying system is not exploited, i.e., $\rho=0$, thus the convergence condition becomes $h(\mu, \rho)=c(k)<0$. In this case, both proposed algorithms reduce to the standard NSAF whose convergence condition is $0<\mu<2$ [4] that makes $c(k)<0$. It is assumed that the step size $\mu$ has been chosen in the range of $0<\mu<2$, then $h(\mu, \rho)$ can be considered as a quadratic function of variable $\rho$, whose discriminant is $g(k)=b^{2}(k)-4 a(k) c(k)$. Evidently, $g(k)>0$ because of $a(k)>0$ and $c(k)<0$, which reveals that $h(\mu, \rho)=0$ has two roots $\rho_{\min }(k)$ and $\rho_{\max }(k)$, as shown in Fig. 2. In other words, we have $\rho_{\min }(k)<\rho<\rho_{\max }(k)$ when $h(\mu, \rho)<0$. Note that $\rho=0$ is also an effective solution of $h(\mu, \rho)<0$, owing to the fact that $h(\mu, 0)=c(k)<0$ for $0<\mu<2$. Also, using negative values of $\rho$ is not in line with the definition of the optimization problems (4) and (9). It follows that $\rho$ should be chosen in the range of $0 \leq \rho<\rho_{\max }(k)=(-b(k)+\sqrt{g(k)}) / 2 a(k)$ to guarantee the mean square stability of both proposed algorithms. It is worthy noting that an optimal value $\rho_{\text {opt }}(k)$ that minimizes $h(\mu, \rho)$ can be solved as $\rho_{\text {opt }}(k)=-b(k) / 2 a(k)>0$, which also implies $b(k)<0, \forall k$.

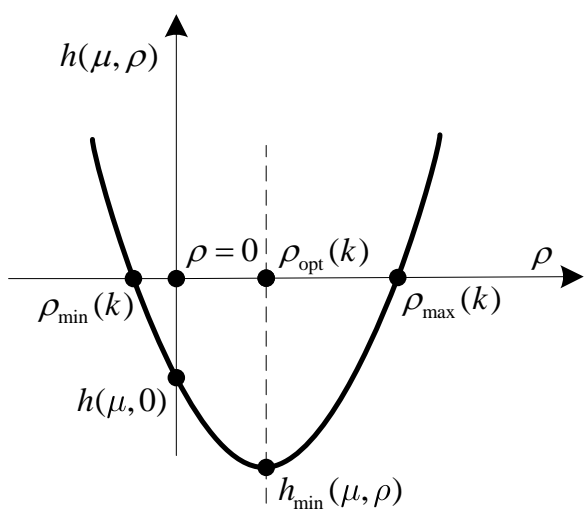

Fig. 2. Curve of the function $h(\mu, \rho)$ versus $\rho$ when $\mu$ is fixed.

\subsection{Superiority of both algorithms}

Assuming that the algorithms have converged to the steady-state, i.e., $\operatorname{MSD}(k+1)=\operatorname{MSD}(k)=\operatorname{MSD}(\infty)$, then $(16)$ becomes

$$
\begin{gathered}
2 E\left[\sum_{i=0}^{N-1} \frac{\tilde{\mathbf{w}}^{T}(\infty) \mathbf{u}_{i}(\infty) e_{i, \mathrm{D}}(\infty)}{\left\|\mathbf{u}_{i}(\infty)\right\|_{2}^{2}}\right]-\mu E\left[\sum_{i=0}^{N-1} \frac{e_{i, \mathrm{D}}^{2}(\infty)}{\left\|\mathbf{u}_{i}(\infty)\right\|_{2}^{2}}\right]=\frac{1}{4} \mu E\left[\left\|\mathbf{P}(\infty) \mathbf{f}_{\beta}(\mathbf{w}(\infty))\right\|^{2}\right] \rho^{2}+ \\
\left\{E\left[\tilde{\mathbf{w}}^{T}(\infty) \mathbf{P}(\infty) \mathbf{f}_{\beta}(\mathbf{w}(\infty))\right]-\mu E\left[\sum_{i=0}^{N-1} \frac{e_{i, \mathrm{D}}(\infty) \mathbf{u}_{i}^{T}(\infty)}{\left\|\mathbf{u}_{i}(\infty)\right\|_{2}^{2}} \mathbf{P}(\infty) \mathbf{f}_{\beta}(\mathbf{w}(\infty))\right]\right\} \rho
\end{gathered}
$$


Substituting (2) and (14) into (18) and applying Assumptions 2-5, then by merging similar terms, we can obtain the steady-state MSD, $\operatorname{MSD}_{\mathrm{I}}(\infty)$, for the ZN-NSAF-I algorithm:

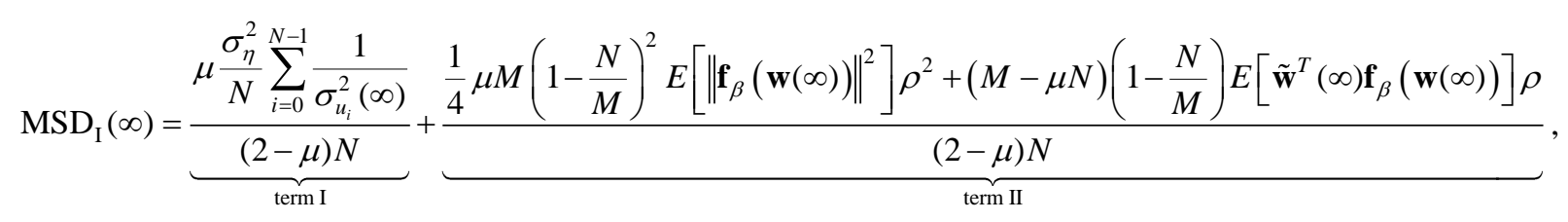

while $\mathrm{MSD}_{\mathrm{II}}(\infty)$ for the ZN-NSAF-II algorithm:

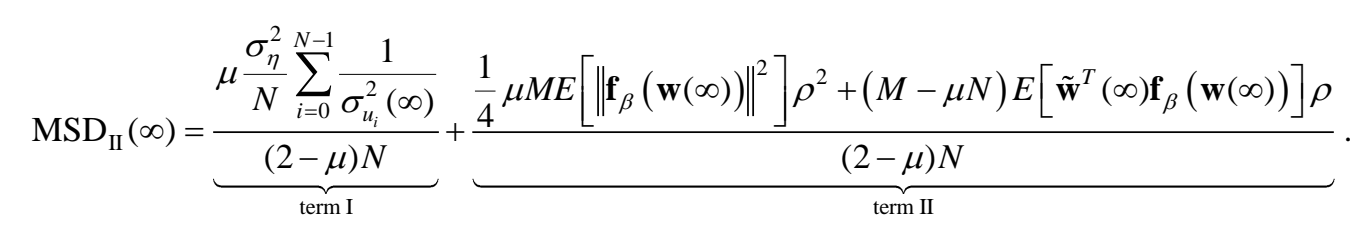

By observing (19) and (20), it is found that they have the same form,

$$
\text { MSD }=\text { 'MSD of the standard NSAF' + 'MSD caused by the } l_{0} \text {-norm attraction' }
$$

where the first term denotes the steady-state MSD of the standard NSAF, and the second term denotes the MSD caused by the $l_{0}$-norm attraction. In other words, the second summands in (19) and (20) are the difference between the MSD of the ZN-NSAF-I and ZN-NSAF-II algorithms and that of the standard NSAF algorithm, respectively, which are denoted as $\phi_{\mathrm{I}}(\rho)$ and $\phi_{\mathrm{II}}(\rho)$. If one appropriately selects $\rho$ to make $\phi_{\mathrm{I}}(\rho)<0$ and $\phi_{\mathrm{II}}(\rho)<0$, both proposed algorithms will outperform the NSAF in the steady-state MSD. To this end, the following inequalities must be hold

$$
\begin{gathered}
0<\rho<\rho_{\mathrm{I}}^{*}=-\frac{4(M-\mu N) E\left[\tilde{\mathbf{w}}^{T}(\infty) \mathbf{f}_{\beta}(\mathbf{w}(\infty))\right]}{\mu(M-N) E\left[\left\|\mathbf{f}_{\beta}(\mathbf{w}(\infty))\right\|^{2}\right]} \text { for the ZN-NSAF-I, } \\
0<\rho<\rho_{\mathrm{II}}^{*}=-\frac{4(M-\mu N) E\left[\tilde{\mathbf{w}}^{T}(\infty) \mathbf{f}_{\beta}(\mathbf{w}(\infty))\right]}{\mu M E\left[\left\|\mathbf{f}_{\beta}(\mathbf{w}(\infty))\right\|^{2}\right]} \text { for the ZN-NSAF-II, }
\end{gathered}
$$

and $E\left[\tilde{\mathbf{w}}^{T}(\infty) \mathbf{f}_{\beta}(\mathbf{w}(\infty))\right]<0$.

Now, if the estimate of a component lies in a small region $w_{m}(\infty) \in[-1 / \beta, 1 / \beta]$, we assume its true value $w_{m, o}=0 \quad$ (i.e., zero components of the unknown vector $\mathbf{w}_{o}$ ). Therefore, according to the definition (12), in steady-state, we get

$$
\begin{gathered}
E\left[\tilde{\mathbf{w}}^{T}(\infty) \mathbf{f}_{\beta}(\mathbf{w}(\infty))\right] \approx \sum \beta^{2} E\left[w_{m}^{2}(\infty)\right]-\beta E\left[\left|w_{m}(\infty)\right|\right], w_{m}(\infty) \in[-1 / \beta, 1 / \beta] \\
E\left[\left\|\mathbf{f}_{\beta}(\mathbf{w}(\infty))\right\|^{2}\right] \approx \sum \beta^{4} E\left[w_{m}^{2}(\infty)\right]-2 \beta^{3} E\left[\left|w_{m}(\infty)\right|\right]+\beta^{2}, w_{m}(\infty) \in[-1 / \beta, 1 / \beta]
\end{gathered}
$$

As shown in (24), the negativeness of $E\left[\tilde{\mathbf{w}}^{T}(\infty) \mathbf{f}_{\beta}(\mathbf{w}(\infty))\right]$ can be always guaranteed.

Remark 3: Although we have provided some closed-form expressions for $\rho_{\mathrm{opt}}(k), \rho^{*}$, and $\rho_{\max }(k)$, these expressions are not practical for choosing $\rho$ in the ZN-NSAF-I and ZN-NSAF-II algorithms, since they depend on the sparse level of the unknown vector. In practice, $\rho$ is a very small positive number (i.e., $0<\rho \ll 1$ ). Even so, the analyses in Section 4 can still give some insights for choosing $\rho$, which are summarized as follows:

1) Both proposed algorithms are mean square stability when $0 \leq \rho<\rho_{\max }(k)$ and $0<\mu<2$.

2) There is a region of $0<\rho<\rho^{*}$ (i.e., $\rho_{\mathrm{I}}^{*}$ and $\rho_{\mathrm{II}}^{*}$ ) that makes both proposed algorithms outperform the NSAF 
algorithm in steady-state performance. Here, we called this region as the superiority region. Also, the optimal $\rho_{\text {opt }}$ for these two algorithms that minimize the MSD are within this region. Notice that there is always $\rho_{\text {opt }}(k)<\rho^{*}<\rho_{\max }(k)$.

3) From (22) and (23), one can find that $\rho_{\mathrm{I}}^{*}$ in the ZN-NSAF-I is slightly larger than $\rho_{\mathrm{II}}^{*}$ in the ZN-NSAF-II.

\section{Simulation results}

To evaluate the performance of the algorithms, simulations are performed in sparse system identification scenarios. The colored input signal is a first-order autoregression, $\operatorname{AR}(1)$, process with a pole at 0.9 . The background noise $\eta(n)$ added at the output of the unknown system is white Gaussian with a signal-to-noise ratio (SNR) of 30dB. In our simulations, a cosine modulated filter bank is used for all the SAF algorithms [2]. The normalized MSD (NMSD), $10 \log _{10}\left(\operatorname{MSD}(k) /\left\|\mathbf{w}_{o}\right\|_{2}^{2}\right),(\mathrm{dB})$, is used as a measure of the performance. All results are obtained by averaging over 30 independent runs.

\subsection{Synthetic sparse system}

In this section, the unknown sparse vector $\mathbf{w}_{o}$ has $M=64$ taps whose nonzero components are randomly set to 1.0 or -1.0 with their positions randomly selected and is normalized by $\mathbf{w}_{o}^{T} \mathbf{w}_{o}=1$ [30], where $S$ denotes the number of nonzero components.

To obtain good performance, the effect of parameters $(\beta, \rho$ and $N$ ) with respect to the performance of the proposed algorithms should be first investigated. In Figs. 3 and 4, we study the performance of the ZN-NSAF-II using different number of subbands (i.e., $N=2,4$, and 8) against different intensities $\rho$. In the simulations, the number of nonzero components is set to $S=4, \rho$ is varied within $\left[3 \times 10^{-7}, 7 \times 10^{-3}\right]$, and we set to $\mu=0.6$ and $\beta=15$ for each algorithm. Fig. 3 shows the steady-state NMSDs of the algorithms, which are obtained by averaging over more than 500 instantaneous NMSD values in steady-state. For illustrating the convergence rate, Fig. 4 depicts the number of the input samples when the algorithms reach $-30 \mathrm{~dB}$ NMSD, where the total number of the input samples is 5000 in the overall adaptive process. As one can see from Figs. 3 and 4, there is a superiority region on $\rho$ that makes the ZN-NSAF-II has smaller steady-state NMSD than the NSAF. And, in this region, the ZN-NSAF-II can faster converge since it requires less input samples than the NSAF for reaching $-30 \mathrm{~dB}$ NMSD. This is because the ZN-NSAF-II exploits the sparsity of the underlying system by means of the $l_{0}$-norm of the weight vector. In addition, as shown in Fig. 4, when $\rho$ is set in the superiority region, the convergence rate of the ZN-NSAF-II with a large number of subbands $N$ is faster than that with a small $N$. This is due to the fact that the decimated subband input signals are closer to white signal for a larger number of subbands. Note that for the ZN-NSAF-II, the increase of $N$ will also damage the steady-state performance. In the following simulations, number of subbands is chosen as $N=4$. 


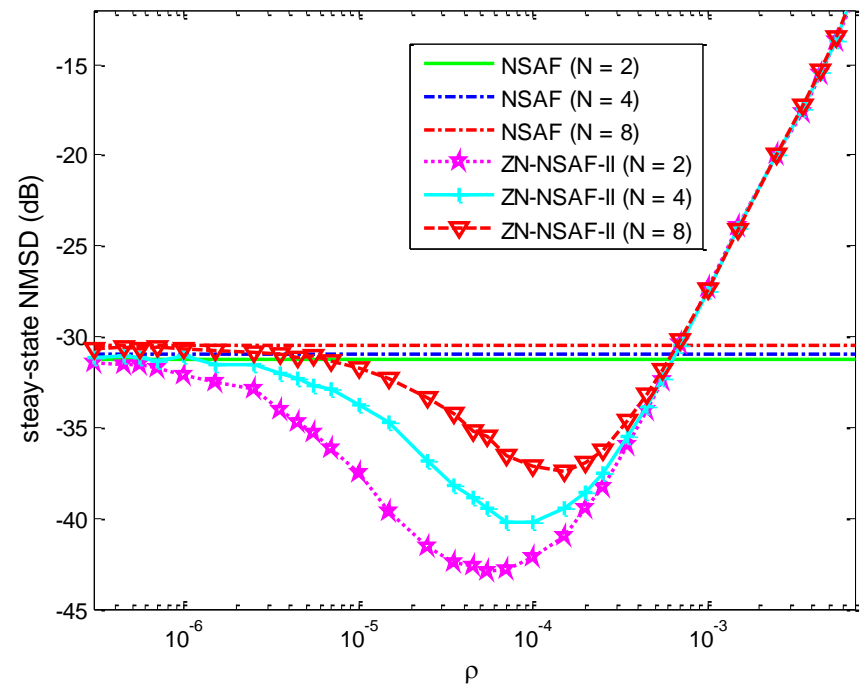

Fig. 3. Steady-state NMSDs of the ZN-NSAF-II using different number of subbands vs intensity $\rho$.

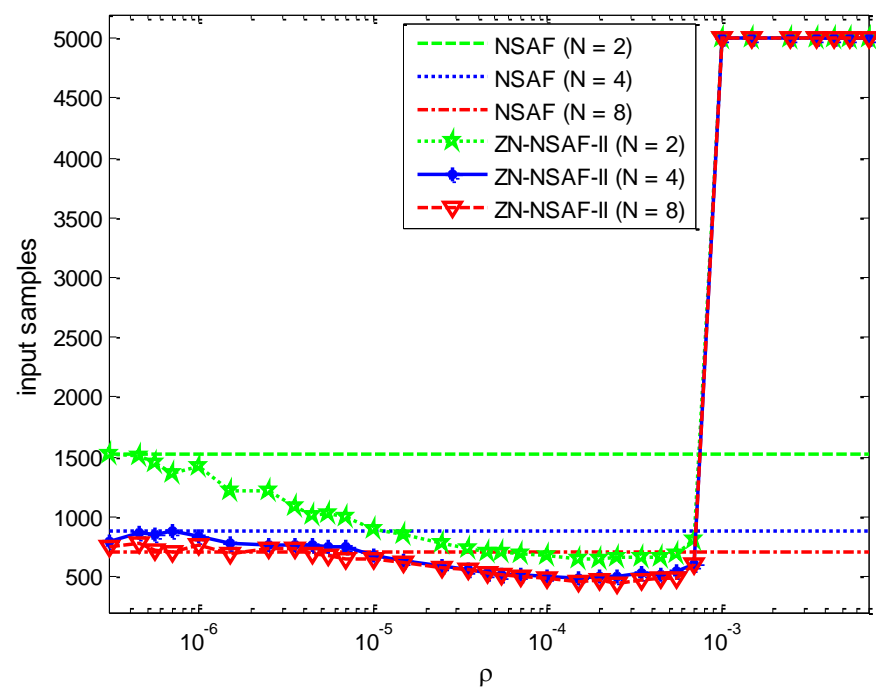

Fig. 4. The number of input samples to reach -30 dB NMSD for the ZN-NSAF-II using different number of subbands vs intensity $\rho$.

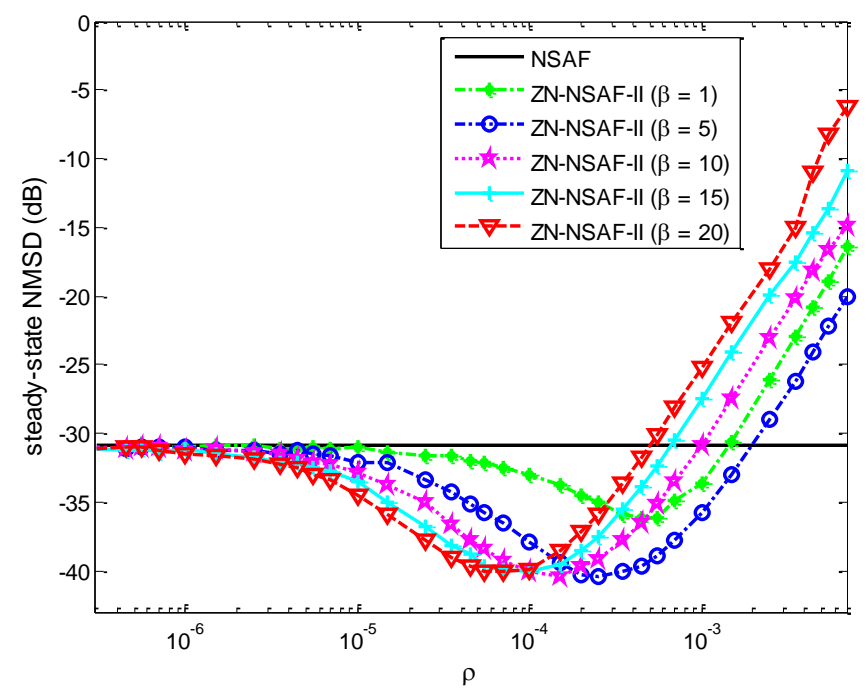

Fig. 5. Steady-state NMSDs of the ZN-NSAF-II using different $\beta$ vs intensity $\rho$.

Fig. 5 depicts the steady-state NMSDs of the ZN-NSAF-II algorithm using different values of $\beta$ against different intensities $\rho$. Simulation setting is the same as Fig. 3. As can be seen, for a given $\beta$ value, there is also a corresponding 
superiority region on $\rho$ so that the ZN-NSAF-II outperforms the NSAF in steady-state performance. And, in this region, with the increasing of $\rho$, the steady-state NMSD of this algorithm is decreased first and then increased after a certain optimal $\rho_{\text {opt }}$. This result is consistent with the conclusions in Remark 3. From Fig. 5, we can know that the proper range of $\beta$ can be determined as $\beta \in[5,20]$.

It has been stated in Section 4 that the superority of both proposed algorithms over the NSAF depend largely on the intensity parameter $\rho$, while it is related to the sparse level of the system to be identified. To this end, Fig. 6 plots the steady-state NMSDs of the ZN-NSAF-II against different intensities $\rho$ in different sparsity scenarios. In this experiment, if the value of $S$ is smaller (e.g., $S<32$ ), then the unknown vector is sparser, conversely, the unknown vector is more dispersive (non-sparse). Other simulation setting is also the same as Fig. 3. As one can see, the superiority of the ZN-NSAF-II will become weak in comparison with the NSAF when the unknown vector becomes dispersive (e.g. $S=48$ ) regardless of values of $\rho$ within the superiority region. The reason is that the coefficients with large values are in the majority in dispersive case, while the role of the zero-attractor is to impose an attraction to zero on small coefficients, see Remark 1. In Figs. 3-6, we only show the results of the ZN-NSAF-II due to its low computational cost, but the conclusions are right for the ZN-NSAF-I since both proposed algorithms have similar performance. In a word, the proposed algorithms are more suitable for sparse cases.

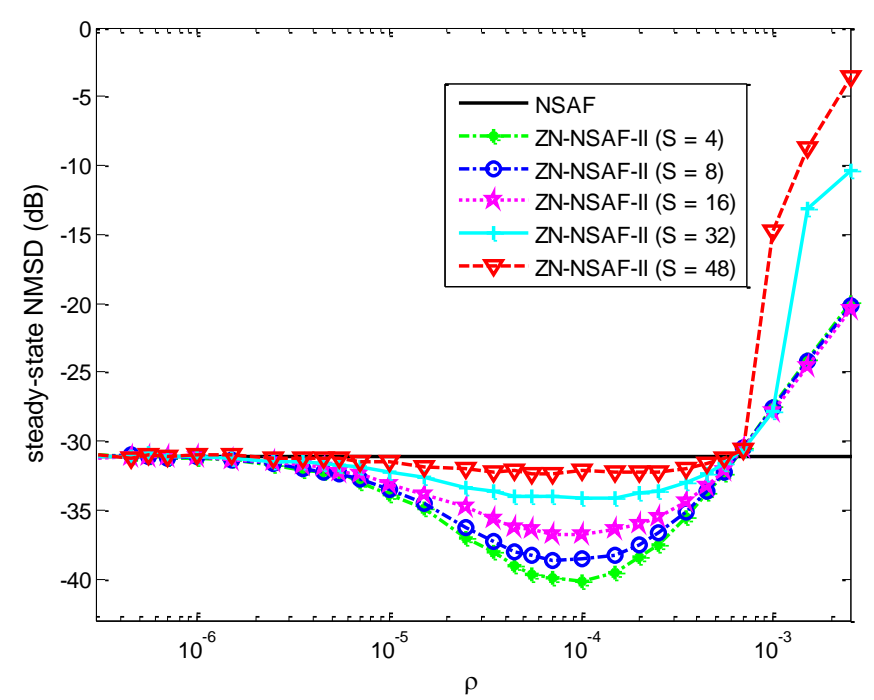

Fig. 6. Steady-state NMSDs of the ZN-NSAF-II vs intensity $\rho$ for different sparse degrees $S . \quad \mathrm{SNR}=30, \mathrm{AR}(1)$ input, and $N=4$.

Next, the performances of both proposed algorithms are compared with the NSAF [4], $l_{1}$-NSAF and $l_{1}$-RNSAF algorithms in [28], as shown in Fig. 7. Here, the number of nonzero components in $\mathbf{w}_{o}$ is changed from $S=4$ to $S=16$ at the $3 \times 10^{3}$ th input samples, and to $S=32$ at the $6 \times 10^{3}$ th input samples. To fairly compare these algorithms, the same step size $\mu=0.6$ is used for all the algorithms, and other parameters are chosen according to their recommended values in the literatures. As can be seen, these sparse algorithms have better performance in term of the convergence rate and steady-state NMSD than the NSAF in a sparse case (e.g., $S=4$ ). As compared to the $l_{1}$-NSAF and $l_{1}$-RNSAF algorithms, both proposed algorithms have smaller steady-state NMSD, because the $l_{0}$-norm can characterize better the sparsity of the underlying system than the $l_{1}$-norm. It is also noticed that as the unknown system becomes less sparse (e.g., $S=32$ ), the superiority of these sparse algorithms over the NSAF will become weak. In addition, both proposed algorithms have almost the same convergence performance while the ZN-NSAF-II has lower computational complexity. 


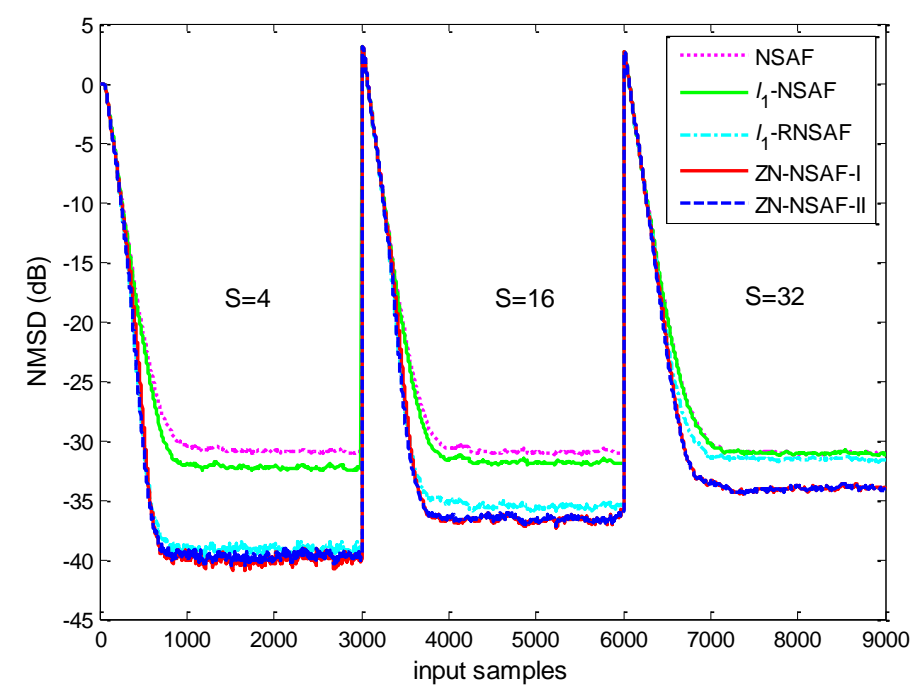

Fig. 7. The NMSD curves of various SAF algorithms for synthetic sparse system. $\gamma=1.5 \times 10^{-6}$ for the $l_{1}-\mathrm{NSAF}$; $\varepsilon=0.01$ and $\gamma=5 \times 10^{-5}$ for the $l_{1}$-RNSAF; $\beta=20$ and $\rho=1 \times 10^{-4}$ for the ZN-NSAF-I and the ZN-NSAF-II.

\subsection{Sparse echo path}

The identification of the echo path is a crucial task in echo cancellation application. In this section, therefore, the unknown vector $\mathbf{w}_{o}$ is a measured acoustic echo path (the sample rate is $8 \mathrm{kHz}$ ) with $M=512$ taps [3]. Also, to evaluate the tracking capability of the algorithms, the echo path changes to $-\mathbf{w}_{o}$ at the $2 \times 10^{4}$ th input samples. Fig. 8 shows the NMSD results of these algorithms. As one can see, both proposed algorithms still exhibit better performance than other SAF algorithms.

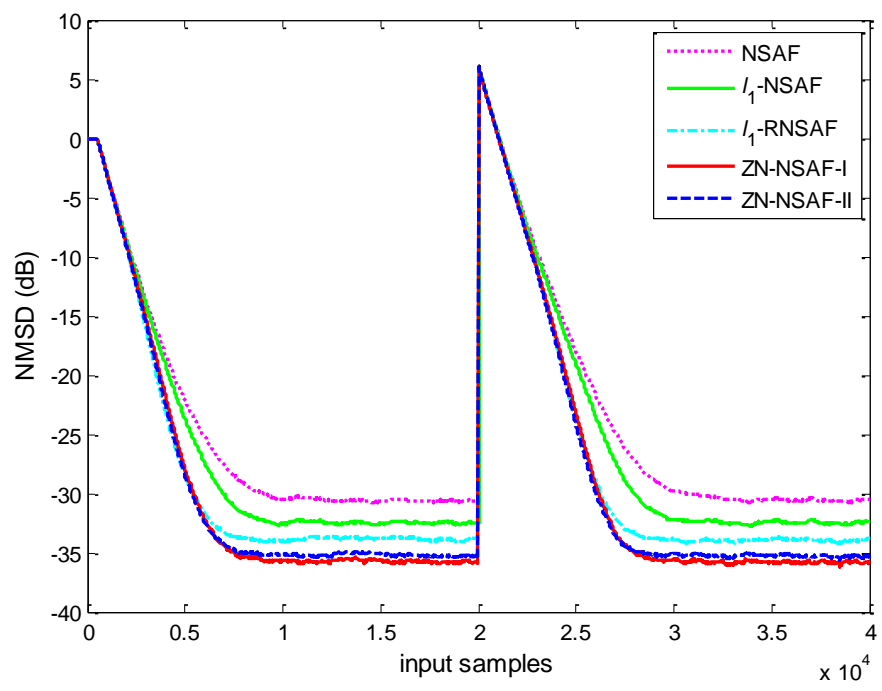

Fig. 8. The NMSD curves of various SAF algorithms for sparse echo path. $\gamma=8 \times 10^{-6}$ for the $l_{1}-\mathrm{NSAF} ; \quad \varepsilon=0.01$ and $\gamma=1 \times 10^{-6}$ for the $l_{1}-\mathrm{RNSAF}$; $\beta=20$ and $\rho=1.3 \times 10^{-6}$ for the ZN-NSAF-I; $\beta=20$ and $\rho=2 \times 10^{-6}$ for the ZN-NSAF-II.

\section{Conclusion}

In this study, by incorporating the $l_{0}$-norm constraint of the weight vector into the optimization formula used for deriving the NSAF, two sparse NSAF algorithms were proposed, i.e., the ZN-NSAF-I and the ZN-NSAF-II. These two algorithms are derived based on the principle of the minimum perturbation and the gradient descent theory, respectively. Then, the convergence of the proposed algorithms and their superiority over the NSAF were analyzed. Simulations have shown that both proposed algorithms work better than their competing algorithms when identifying sparse systems. Interestingly, these two algorithms have similar performance, but the ZN-NSAF-II is preferred in practice due to its low computational complexity. 


\section{Acknowledgment}

This work was partially supported by National Science Foundation of P.R. China (Grant: 61271340, 61571374 and 61433011) and supported in part by 973 Program (No. 2015CB351703).

\section{References}

[1] H. Sayed, Fundamentals of Adaptive Filtering. New York:Wiley, 2003.

[2] K. A. Lee, W. S. Gan, and S. M. Kuo, Subband adaptive filtering: theory and implementation. Hoboken, NJ: Wiley, 2009.

[3] H. Zhao, Y. Yu, S. Gao, X. Zeng, and Z. He, "Memory proportionate APA with individual activation factors for acoustic echo cancellation,” IEEE/ACM IEEE Trans. Audio, Speech, Lang. Process., vol. 22, no. 6, pp. 1047-1055, 2014.

[4] K. A. Lee, and W. S. Gan, "Improving convergence of the NLMS algorithm using constrained subband updates," IEEE Signal Process. Lett., vol. 11, no. 9, pp. 736-739, 2004.

[5] K. A. Lee, W. S. Gan, and S. M. Kuo, "Mean-square performance analysis of the normalized subband adaptive filter," in Proc. 40th Asilomar Conf. Signals, Syst., Comput., 2006, pp. 248-252.

[6] W. Yin and A. S. Mehr, "Stochastic analysis of the normalized subband adaptive filter algorithm," IEEE Trans. Circuits Syst. I, vol. 58, no. 5, pp. 1020-1033, 2011.

[7] K.A. Lee and W.S. Gan, "Inherent decorrelating and least perturbation properties of the normalized subband adaptive filter," IEEE Trans. Signal Process., vol. 54, no. 11, pp. 4475-4480, 2006.

[8] J. Ni, and F. Li, “A variable step-size matrix normalized subband adaptive filter," IEEE Trans. Audio Speech Lang. Process., vol. 18, no. 6, pp. 1290-1299, 2010.

[9] J.J. Jeong, K. Koo, G.T. Choi, and S.W. Kim, “A variable step size for normalized subband adaptive filters,” IEEE Signal Process. Lett. vol. 19, no. 12, pp. 906-909, 2012.

[10] Y. Yu, H. Zhao, Z. He, and B. Chen, “A robust band-dependent variable step size NSAF algorithm against impulsive noises,” Signal Process., vol. 119, pp. 203-208, 2016.

[11] J. Radecki, Z. Zilic, and K. Radecka, "Echo cancellation in IP networks,” in Proc.45th Midwest Symp. Circuits Syst., 2002, vol. 2, pp. $219-222$.

[12] W. F. Schreiber, “Advanced television systems for terrestrial broadcasting: Some problems and some proposed solutions," in Proc. IEEE, vol. 83, pp. 958-981, 1995.

[13] D. L. Duttweiler, "Proportionate normalized least mean squares adaptation in echo cancelers," IEEE Trans. Speech Audio Process., vol. 8, no. 5, pp. $508-518,2000$.

[14] J. Benesty and S. L. Gay, “An improved PNLMS algorithm,” in Proc. IEEE Int. Conf. Acoust., Speech, Signal Process. (ICASSP’02), 2002, vol. 2, pp. 1881-1884.

[15] H. Deng and M. Doroslovacki, "Improving convergence of the PNLMS algorithm for sparse impulse response identification," IEEE Signal Process. Lett., vol. 12, no. 3, pp. 181-184, 2005.

[16] L. Liu, M. Fukumoto, and S. Saiki, “An improved $\mu$-law proportionate NLMS algorithm,” in Proc. IEEE Int. Conf. Acoust., Speech, Signal Process. (ICASSP'08), 2008, pp. 3797-3800.

[17] M. S. E. Abadi, S. Kadkhodazadeh “A family of proportionate normalized subband adaptive filter algorithms,” Journal of the Franklin Institute, vol. 348, no. 2, pp. 212-238, 2011.

[18] R. Tibshirani, "Regression shrinkage and selection via the LASSO,” J. Royal. Statist. Soc B., vol. 58, pp. $267-288,1996$.

[19] R. Baraniuk, “Compressive sensing,” IEEE Signal Processing Magazine, vol. 25, pp. 21-30, 2007.

[20] Y. Chen, Y. Gu, and A. O. Hero, “Sparse LMS for identification,” in Proc. IEEE Int. Conf. Acoust., Speech, Signal Process. (ICASSP’09), Taipei, 2009, pp. 3125-3128.

[21] Y. Gu, J. Jin, and S. Mei, “ $l_{0}$-norm constraint LMS algorithm for sparse system identification,” IEEE Signal Process. Lett., vol. 16, no. 9, pp. 774-777, 2009.

[22] G. L. Su, J. Jin, Y. Gu, and J. Wang, "Performance analysis of $l_{0}$-norm constraint least mean square algorithm,” IEEE Trans. Signal Process., vol. 60, no. 5, pp. 2223-2235, 2012.

[23] R. Meng, R.C. de Lamare, and V.H. Nascimento, "Sparsity aware affine projection adaptive algorithms for system identification," in Sensor Signal Processing for Defence (SSPD 2011), London, U.K., 2011, pp. 1-5.

[24] M. V. S. Lima, W. A. Martins, and P. S. R. Diniz, “Affine projection algorithms for sparse system identification,” in Proc. IEEE Int. Conf. Acoust., Speech, Signal Process. (ICASSP’13), Vancouver, BC, pp. 5666-5670, 2013.

[25] E.M. Eksioglu and A.K. Tanc, "RLS algorithm with convex regularization,” IEEE Signal Process. Lett., vol. 18, no. 8, pp. 470-473, 2011.

[26] E.M. Eksioglu, "Sparsity regularized RLS adaptive filtering,” IET Signal Process., vol. 5, no. 5, pp. 480-487, 2011.

[27] Y. Liu, C. Li, and Z. Zhang, "Diffusion sparse least-mean squares over networks,” IEEE Trans. Signal Process., vol. 60, no. 8, pp. 4480-4485, 2012. 
[28] Y. S. Choi, "Subband adaptive filtering with $l_{1}$-norm constraint for sparse system identification," Mathematical Problems in Engineering, vol. 2013, 7 pages, 2013. doi:10.1155/2013/601623.

[29] Taheri, and S. A. Vorobyov, "Reweighted $l_{1}$-norm penalized LMS for sparse channel estimation and its analysis," Signal Processing, vol. 104, pp. 70-79, 2014.

[30] F. Wu and F. Tong, "Gradient optimization p-norm-like constraint LMS algorithm for sparse system estimation,” Signal Processing, vol. 93, pp. 967-971, 2013.

[31] F. Wu, Y. Zhou, F. Tong and R. Kastner, "Simplified p-norm-like constraint LMS algorithm for efficient estimation of underwater acoustic channels," Journal of Marine Science and Application, vol. 12, no. 2, pp. 228-234, 2013.

[32] D. Yin, H. C. So, and Y. Gu, "Sparse constraint affine projection algorithm with parallel implementation and application in compressive sensing," in Proc. IEEE Int. Conf. Acoust., Speech, Signal Process. ICASSP, 2014, pp. 7238-7242.

[33] B. Chen, L. Xing, H. Zhao, N. Zheng, and J. C. Principe, "Generalized correntropy for robust adaptive filtering," IEEE Trans. Signal Process., vol. 64, no. 13, pp. 3376-3387, 2016.

[34] B. Chen, L. Xing, J. Liang, N. Zheng, J. C. Principe, "Steady-state mean-square error analysis for adaptive filtering under the maximum correntropy criterion,” IEEE Signal Process. Lett., vol. 21 , no. 7, pp. 880-884, 2014.

[35] Z. Wu, S. Peng, B. Chen, and H. Zhao, "Robust hammerstein adaptive filtering under maximum correntropy criterion,” Entropy, vol. 17, no. 10, pp. 7149-7166, 2015

[36] B. Chen, J. Wang, H. Zhao, N. Zheng, and J. C. Principe, "Convergence of a fixed-point algorithm under maximum correntropy criterion,” IEEE Signal Processing Letters, vol. 22, no. 10, pp. 1723-1727, 2015. 